\title{
Norois
}

Environnement, aménagement, société

$223 \mid 2012$

Villes petites et moyennes

\section{Les migrations de médecins roumains vers la France, entre démographie médicale et quête de meilleures conditions d'exercice}

The Romanian doctors' migrations to France: between demographical crisis and hope of better practice conditions

\section{Raymonde Séchet et Despina Vasilcu}

\section{OpenEdition \\ Journals}

Édition électronique

URL : https://journals.openedition.org/norois/4203

DOI : 10.4000/norois.4203

ISBN : 978-2-7535-2043-1

ISSN : 1760-8546

Éditeur

Presses universitaires de Rennes

Édition imprimée

Date de publication : 30 mars 2012

Pagination : 63-76

ISBN : 978-2-7535-2041-7

ISSN : 0029-182X

Référence électronique

Raymonde Séchet et Despina Vasilcu, « Les migrations de médecins roumains vers la France, entre démographie médicale et quête de meilleures conditions d'exercice », Norois [En ligne], 223 | 2012, mis en ligne le 28 février 2014, consulté le 13 janvier 2022. URL : http://journals.openedition.org/norois/ 4203 ; DOI : https://doi.org/10.4000/norois.4203 


\title{
Les migrations de médecins roumains vers la France, entre démogra- phie médicale et quête de meilleures conditions d'exercice
}

\author{
The Romanian Doctors' Migrations to France: \\ Between Demographical Crisis and Hope of Better Practice Conditions
}

Raymonde SÉCHET ${ }^{\mathrm{a} *}$, Despina VASILCu ${ }^{\mathrm{b}}$

* auteur correspondant

a Université Européenne de Bretagne, Université Rennes 2, CNRS, ESO, UMR 6590F - 35000 Rennes, France

(raymonde.sechet@univ-rennes2.fr)

${ }^{\text {b }}$ Département de géographie, Université « Stefan cel Mare » de Suceava, Roumanie - Strada Universitatii, nr. 13, code 720229

Résumé : Les professionnels de santé roumains sont nombreux à partir exercer à l'étranger. En France, leur présence contribue à atténuer les problèmes de démographie médicale. En revanche, cet exode aggrave les difficultés du système sanitaire roumain. Ces migrations s'inscrivent dans un marché mondial des personnels médicaux en cours de constitution défavorable à la Roumanie comme à beaucoup de pays en développement ou en transition. Les incitations de l'Organisation Mondiale de la Santé pour une éthique des politiques migratoires ne sont pas suffisantes pour réguler les flux.

Abstract: Many Romanians doctors leave their country to work abroad. In France, Romanian doctors contribute to alleviate the problems of medical demography, ie the lack of doctors in many remote rural areas, and also in hospitals and in some medical specialities. On the other hand, these migrations increase difficulties for the provision of medical services in Romania. These migrations take place in the current development of a global market for medical personnel which has negative consequences in many developing countries. The WHO recommendations in migratory policies are limited to the scale of ethics and are not enough to enable a regulation of these migration flows.

Mots clé : migration internationale des élites - démographie médicale - médecins - Roumanie - Union européenne - France

Keywords: international elite migration - medical demography - physicians - Romania - European Union - France

\section{INTRODUCTION}

Les mobilités internationales actuelles de médecins s'inscrivent dans le contexte de déficit de personnel médical dans les pays d'Europe occiden- tale et d'élargissement d'un marché mondial des compétences médicales qui est plus complexe que celui des années 1980. À cette époque, de nombreux médecins ont, à l'image des scientifiques ori- 
ginaires tant d'Europe occidentale que du reste du monde, été attirés par les conditions de rémunération offertes aux États-Unis. Et les départs étaient d'autant plus aisés que les Etats-Unis avaient mis en place des politiques structurées et cohérentes visant à favoriser l'immigration de personnels de haut niveau. En fait, après l'Australie et le Canada, qui ont été les premiers à ouvrir la course au personnel médical formé en dehors de leurs frontières, puis les États-Unis, les pays européens sont à leur tour entrés sur le marché mondial des compétences médicales quand ils ont été confrontés au manque de professionnels de la santé. En France, ce manque a été reconnu à la fin des années 1990 sous l'expression « crise de la démographie médicale ».

Dans le cadre de l'émergence d'un marché du travail européen découlant de la mise en place d'une politique migratoire communautaire harmonisée (Weber, 2009) et dans lequel l'immigration est supposée jouer un rôle décisif, la plupart des pays de l'Union européenne ont pris des mesures visant à faciliter la venue de travailleurs qualifiés et très qualifiés, tout en cherchant à empêcher l'immigration de travailleurs peu qualifiés. Après avoir sévèrement critiqué les États-Unis, les pays d'Europe occidentale ont à leur tour, et de manière convergente, adopté des mesures d'immigration sélective (Weber, 2007). En Europe, les déplacements de personnels médicaux vers l'Ouest se sont dès lors intensifiés, au point qu'après 2004 et 2007 les pays concernés ont, à leur tour, été accusés de piller les compétences en provenance des nouveaux États membres de l'Union européenne.

Les premières initiatives pour favoriser l'immigration de personnes représentant un capital humain élevé sont celles qui ont été prévues dans le cadre des politiques d'immigration menées par l'Allemagne en 2000 pour le recrutement à l'étranger d'environ 20000 informaticiens, par l'Autriche en 2003, par le Royaume-Uni qui a lancé en 2002 le « Highly Skilled Migrant Program » (Tandonnet, 2003 ; Salt, 2005). En France, même si cela n'a pas été explicitement reconnu, les dispositifs en faveur de l'immigration choisie, symbolisés par la carte "Compétences et Talents », ont eu pour objectif de favoriser l'immigration d'élites, dont celle des médecins.

Au $1^{\text {er }}$ janvier 2009, près de 10000 médecins étrangers exerçaient en France, parmi lesquels les médecins roumains arrivaient au deuxième rang (CNOM, 2009). Pourquoi les médecins roumains sont-ils si nombreux à venir en France, à quels besoins répondent-ils et quels sont les effets de leur départ pour la Roumanie? Si, pour des migrations de travail aussi spécifiques que celle des médecins roumains, l'analyse classique en termes de facteurs d'attraction et de répulsion peut sembler de prime abord pertinente tant les écarts de revenus au sein de l'UE sont importants, elle s'avère en fait notoirement insuffisante dès lors que l'on cherche à passer de la description des flux à la compréhension des processus. Les phénomènes en jeu sont complexes et combinent des éléments relatifs aux parcours personnels dans le contexte spécifique de la Roumanie contemporaine, à la demande de personnel soignant en Europe de l'Ouest, à la diffusion des modèles professionnels, sociaux et culturels et à la levée progressive des obstacles juridiques à la mobilité professionnelle au sein de l'UE, à la force des réseaux.

Après une synthèse des facteurs qui ont favorisé l'immigration de médecins roumains, on s'intéressera plus particulièrement aux caractéristiques des personnels médicaux roumains installés en France avant de poser quelques questions relatives aux conséquences de ces migrations pour la Roumanie. Pour cela, on s'appuiera sur les résultats d'une enquête menée au printemps 2010 auprès de médecins roumains exerçant dans l'Ouest de la France et en Île-de-France, complétée, pour la France, par des données nationales (Ministère de la santé et Conseil national de l'ordre des médecins) et, pour la Roumanie, par une revue de littérature. L'enquête par entretiens semi-directifs a concerné 106 médecins roumains. La passation sur le lieu d'exercice a facilité les rencontres mais limité la durée des entretiens. Ceux-ci ont donc porté avant tout sur les parcours professionnels en Roumanie et en France, de la fin des études à la situation au moment de l'entretien, et sur les conditions de la migration.

\section{UNE CONVERGENCE DE FACTEURS FAVORABLES À LA MIGRATION DES MÉDECINS ROUMAINS}

La croissance des flux migratoires en direction des pays développés découle de la convergence de décisions individuelles qui dépendent de la rencontre entre des motivations et des aspirations 
personnelles et professionnelles et un ensemble de facteurs d'ordre politique, économique, social, démographique. Pour le cas particulier des migrations de médecins roumains, il s'agit, entre autres, du contexte de crise de la démographie médicale française, de difficultés sociales et professionnelles en Roumanie et d'élargissement de l'Union européenne.

\section{La voie ouverte par l'élargissement de l'union européenne}

L'élargissement progressif de l'Union européenne a ouvert la voie à une forte hausse des migrations internes qui a modifié le paysage migratoire européen. Tout en contribuant au durcissement des dispositifs de protection des frontières de l'espace Schengen, les pays de l'Europe de l'Ouest ont mis à profit l'extension de la zone de libre circulation pour étendre vers l'Est leur espace de recrutement de compétences. Les actifs hautement qualifiés de Roumanie et de Bulgarie, dont les médecins, ont pu bénéficier de l'entrée de leur pays dans l'Union européenne en 2007 et, dès lors, contribuer à la dynamique d'intensification des migrations de travailleurs hautement qualifiés.

La reconnaissance des qualifications professionnelles permise par la directive 2005/36/CE du Parlement européen et du Conseil du 7 septembre 2005 a rendu possible la libre circulation des travailleurs entre tous les pays membres de l'Union européenne. En créant les conditions favorables à l'émergence d'un marché européen des professionnels de santé, cette mesure a autorisé l'accès à « la voie royale des migrations » (Tandonnet, 2003). Nombre de médecins roumains déployaient déjà de véritables stratégies individuelles pour valoriser leurs compétences : ils avaient anticipé l'entrée de leur pays dans l'Union européenne et la reconnaissance de leurs diplômes, notamment en venant faire leur spécialisation au sein des hôpitaux français (la possibilité d'exercer en France pour les étrangers titulaires de l'un des diplômes sanctionnant une formation de spécialiste a été octroyée par un arrêté du 23 mars 2000). La préparation du diplôme interuniversitaire de spécialisation [DIS] était, pour beaucoup de jeunes médecins roumains, un moyen de se faire apprécier, avec l'espoir d'être employé à l'issue de la période de spécialisation. Les effectifs de médecins roumains présents en France avaient donc déjà fortement augmenté depuis le début des années 2000 (Cash et Ullman, 2008). C'est toutefois en 2007 qu'est intervenue la vague la plus forte, constituée d'ailleurs non seulement des médecins mais aussi d'autres catégories de personnes qualifiées roumaines : alors qu'en 2004 la Roumanie arrivait en huitième position des pays pour le nombre de bénéficiaires de titres de séjour professionnels, avec 496 titres, en 2007, elle devance tous les autres pays (SGCICI, 2008).

\section{En France, relever le défi de la démographique médicale}

En même temps qu'augmentait le nombre de médecins roumains présents en France, celle-ci devenait la première destination pour les médecins roumains candidats au départ. C'est que la France était confrontée à la peur de manquer de médecins.

Cette crainte a émergé au début des années 2000, au point qu'un Observatoire national de la démographie médicale a été créé en 2003. Les auteurs des projections établies en 2002 ont affirmé que, sous l'effet conjugué du vieillissement des médecins et des mesures de numerus clausus qui ont limité les effectifs d'étudiants, le nombre de médecins baisserait fortement à l'horizon 2020; alors qu'elles avaient progressé de 263 médecins pour 100000 habitants en 1980 à 329 en 2000, les densités médicales n'auraient plus été que de 307 en 2010 et de 250 en 2020 (Darriné, 2002). Créé en 1971 et stable jusqu'en 1983, avec environ 8000 places par an, le numerus clausus a ensuite été réduit pour atteindre son niveau le plus bas en 1993 (3 500 places) et rester faible ensuite (4 700 places en 2002, année de la prise de conscience réelle des risques de pénurie). Comme il faut près de 10 ans pour former un médecin, ce sont, dans les années 2000, des cohortes peu nombreuses qui sont entrées en activité à un moment où les médecins appartenant aux générations de baby boomers arrivaient à l'âge de la cessation d'activité. Pour les mêmes raisons de faiblesse des numerus clausus jusqu'au milieu de la décennie 2000 et d'âge des praticiens (en 2006, plus de la moitié des médecins ont plus de 50 ans), la poursuite de la baisse paraissait inéluctable pour la décennie 2010 (Attal-Toubert et Vanderschelden, 2009). En 2007, l'Observatoire national des emplois 
et des métiers de la Fonction publique hospitalière a d'ailleurs fait savoir qu'en dix ans environ 75000 médecins de la génération des baby-boomers arriveraient à l'âge de la retraite et que cela représentait le double des effectifs susceptibles de les remplacer. Or, dans le même temps, la population française dans son ensemble a connu un processus de vieillissement et donc une croissance des besoins et de la demande de soins.

Les densités médicales nationales sont, en fait, un indicateur très grossier de l'adéquation entre offre et demande de soins puisqu'il ne tient pas compte de l'intensité variable des besoins. Cela est d'autant plus vrai que l'offre est très inégalement répartie, que les nouveaux professionnels rechignent à s'installer en libéral en milieu rural ou dans les zones urbaines défavorisées ou à exercer dans les hôpitaux de pôles urbains secondaires, que les déficits sont particulièrement marqués dans certaines spécialités, dont la psychiatrie, et que les étudiants boudent la médecine générale : en 2004, un tiers des places offertes par l'examen national classant de fin de deuxième cycle n'ont pas été affectées (Bessière et al., 2004).

En conséquence, le recours à des médecins étrangers est bel et bien une opportunité pour répondre à court terme aux problèmes de démographie médicale, au moins tant qu'un véritable relèvement des numerus clausus n'aura pas porté ses fruits, et cela même si, dans les projections ministérielles, les flux internationaux de médecins sont supposés nuls (Attal-Toubert et Vanderschelden, 2009). Avec le recul, la baisse du numerus clausus que ses promoteurs ont justifiée par l'argument que la réduction de l'offre de soin réduirait la demande et aurait pour effet de réduire les dépenses de santé peut, au final, être interprétée comme une mesure technique adoptée dans le cadre idéologique d'érection de la santé en marché, et ayant eu pour effet de générer une propension à une demande de main-d'œuvre venue de l'étranger et de donner naissance à un couple migratoire spécifique.

\section{En Roumanie, des facteurs qui incitent à la migration des médecins}

Un ensemble de facteurs a en effet incité les professionnels de santé roumains à quitter leur pays pour aller exercer en France. Leur migration peut être appréhendée comme une stratégie d'adaptation à la nouvelle situation économique du pays. À la différence des migrations illégales et à l'errance d'actifs peu qualifiés confrontés à la crise des industries roumaines qui a fait suite à la chute du régime Ceausescu en 1989, la migration des médecins provient du désir de quitter un pays qui, pensent-ils, ne leur offre ni le niveau de reconnaissance espéré ni de véritables perspectives professionnelles. Partir est une forme de résistance personnelle face à l'absence d'avenir ou de possibilité de réalisation de soi (Tandonnet, 2003).

L'ampleur prise par les migrations de médecins est à la mesure de l'effondrement de l'économie et de la dégradation du niveau de vie en Roumanie. La précarité de l'équilibre économique du pays influe négativement sur le système de soins. L'écart entre les dépenses de santé de la Roumanie et celles des pays à haut niveau de revenu est un indicateur des inégalités du potentiel d'accès aux soins des populations. En 2008, la Roumanie n'a consacré que 4,5\% de son PIB aux dépenses de santé, soit la moitié du niveau moyen des dépenses des Etats membres de l'UE (9\% du PIB), la France ayant la même année consacré 11,2\% de son PIB aux dépenses de santé. La situation s'est encore dégradée puisque qu'en 2010 le gouvernement roumain a annoncé que seulement 2,6\% du PIB seraient consacrés à la santé et qu'au $1^{\text {er }}$ juillet de la même année les salaires des professionnels de santé ont été réduits de $25 \%$.

Même si des facteurs non-économiques peuvent intervenir dans les décisions d'émigrer, celles-ci ont souvent pour base des raisons financières. Les motivations économiques ont été invoquées comme premier facteur de départ par $70 \%$ des personnes enquêtées en 2010. Ce résultat converge avec ceux d'autres études sur les migrations de travailleurs hautement qualifiés pour confirmer que la perception des écarts de niveau de vie selon les pays augmente avec le niveau de diplôme (Gaillard et Gaillard, 1999; Mayer, 2009; Cortès et Faret, 2009; Docquier et Rapoport, 2007). À la différence d'autres catégories de travailleurs roumains émigrés qui ont été confrontés à la difficulté de trouver un emploi, les médecins émigrés en France ont pu négocier leurs diplômes et bénéficier de facilités pour organiser leur venue. Ils ont souvent bénéficié du soutien des acteurs impliqués dans leur recrutement et intéressés au comblement du déficit de per- 
sonnel médical en France, et donc enclins à favoriser leur adaptation et leur intégration. Plus du quart des médecins enquêtés ont déclaré avoir bénéficié d'un soutien continu de la part des acteurs locaux, qu'il s'agisse de directeurs d'hôpital ou de municipalités dans le cas des médecins généralistes, et $30 \%$ ont déclaré avoir reçu une aide matérielle (logement gratuit, mise à disposition de locaux professionnels, versement d'une prime d'installation, exonérations fiscales), en contradiction avec les principes de la libre installation des professions libérales en France mais en accord avec le principe républicain d'égalité et de solidarité territoriale.

Avec un système de santé qui n'assure pas des conditions favorables de travail et de soins, les médecins roumains ont le sentiment d'être dans l'impossibilité d'exercer pleinement et correctement leurs obligations vis-à-vis des patients, ce qui porte atteinte au sentiment de satisfaction professionnelle et à l'estime de soi. Les représentations du système médical français supposé offrir des équipements et des matériels médicaux modernes, favorables à la sécurité de l'acte médical dans un système de santé bien financé ainsi qu'un milieu professionnel stimulant ont été d'autres facteurs incitatifs.

À ces opportunités professionnelles favorables à l'émigration des médecins s'ajoutent des facteurs personnels : qualité de vie, meilleures opportunités d'éducation et de formation pour les enfants. Les femmes, qui représentent $70 \%$ des médecins roumains exerçant en France, sont les plus sensibles à ces facteurs personnels. Même si quelques unes des femmes enquêtées sont venues sans leurs enfants, la majorité ont entraîné toute leur famille dans la migration, avec d'emblée le souhait d'une installation de longue durée ou même définitive, ne serait-ce qu'en raison de la difficulté à faire valoir leur émancipation en cas de retour : la migration est créatrice d'un espace d'autonomisation féminine (Potot, 2003; Simon, 2008).

Pour les médecins roumains, hommes et femmes qui aspirent à partir exercer à l'étranger, des facteurs culturels et historiques jouent en faveur de la France : même si l'anglais gagne du terrain, le français est largement enseigné en Roumanie et garde son rôle de langue culturelle. Leur niveau de mâ̂trise de la langue française au moment de l'arrivée en France a d'ailleurs été considéré comme élevé par $44 \%$ des personnes enquêtées et moyen par
$40 \%$. Cette maîtrise du français facilite l'intégration professionnelle et sociale. Les conditions générales de vie en Roumanie entrent également en ligne de compte parmi les facteurs ayant conduit à la décision de partir. La crise politique, la corruption, la méfiance envers les institutions de l'Etat, la détérioration des valeurs de la démocratie ont été cités comme des facteurs ayant poussé à quitter le pays au profit de la France.

\section{LES MÉDECINS ROUMAINS DANS LE PAYSAGE MÉdicAL FRANÇAIS}

\section{Une présence croissante}

Le nombre de médecins roumains inscrits au Tableau de l'Ordre des médecins français a fortement augmenté au cours des dernières années, passant de 158 au $1^{\text {er }}$ janvier 2007 à 819 au $1^{\text {er }}$ janvier 2008 et

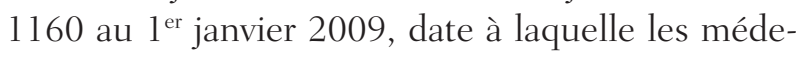
cins roumains arrivent au deuxième rang des médecins étrangers exerçant en France (12\% contre $16 \%$ pour les médecins belges). En 2008, près de $40 \%$ des nouveaux inscrits étrangers étaient des Roumains (CNOM, 2009). Ce pic d'immigration récente s'observe dans la population que nous avons enquêtée, avec $11 \%$ d'arrivées en 2007, $15 \%$ en 2008 et $19 \%$ pour 2009 et le début de l'année 2010. Cependant, ces médecins roumains arrivés récemment sont moins nombreux que ceux qui se sont installés avant 2007, année de la reconnaissance officielle des diplômes obtenus en Roumanie (45\% contre $55 \%$ pour la France entière, $42 \%$ contre $58 \%$ pour la Bretagne). Beaucoup de ceux qui sont venus avant cette date l'ont fait dans le cadre d'une formation à une spécialité, suite à laquelle ils sont restés.

De fait, après le décret n 90-97 du 25 janvier 1990 puis l'arrêté de mars 2000 qui ont établi les conditions d'accès aux formations spécialisées du troisième cycle des études médicales pour les médecins étrangers autres que les ressortissants d'Etats appartenant aux communautés européennes, les hôpitaux français ont fait appel à des praticiens étrangers. Les Roumains venus dans ce cadre ont été peu visibles face aux effectifs alors beaucoup plus nombreux de praticiens venus du Maghreb et du Proche-Orient. Ils ont pourtant contribué à la venue des vagues récentes. 
Bien que l'importance des réseaux sociaux dans les migrations est discutée (Faret, 2003; Hily et al., 2004), l'enquête réalisée en 2010 a cherché à en mesurer l'importance pour les médecins roumains. Il semble bien que les médecins roumains déjà présents en France ont favorisé la venue des suivants : de petits noyaux diasporiques ont soutenu l'arrivée de leurs proches, abaissant ainsi le coût économique, social et culturel de l'installation des nouveaux arrivants. Près de $10 \%$ des médecins enquêtés ont déclaré que leur venue en France a été favorisée par la présence antérieure de collègues et amis. L'arrivée des médecins roumains a également été facilitée par l'action des agences de recrutement chargées de mettre en pratique la politique active d'immigration menée par les pays occidentaux. Citons l'ARIME (Association pour la recherche et l'installation des médecins européens), Revitalis (agence française spécialisée dans le recrutement des médecins pour le milieu rural), Pluri Consultants Roumanie, P\&P Conseil, Paragona (société de recrutement fondée en 2002, actuellement leader sur le marché européen et qui recrute pour la Suède, le Danemark, la Norvège, la GrandeBretagne et la France), mais aussi des agences régionales de développement local comme l'ARDTA (Agence régionale de développement des territoires d'Auvergne), même si elle recrute davantage des personnels de soins que des médecins. Parmi les médecins enquêtés en Bretagne, $15 \%$ sont venus en France par l'intermédiaire de l'une ou l'autre des trois premières.

Des hôpitaux ou des collectivités locales engagent des sommes significatives pour que ces agences trouvent le spécialiste ou le généraliste indispensable à l'équipe médicale de l'établissement ou à la survie du cabinet libéral. Pour autant, les médecins roumains ne s'installent pas forcément là où ils sont les plus attendus, selon une vision dualiste du marché du travail médical qui concentrerait les médecins à diplômes étrangers dans les espaces, les lieux d'exercice et les spécialités délaissées par les médecins nationaux.

En 2009, les médecins roumains sont surtout nombreux dans le Nord de la France, le Nordest (Alsace, Lorraine, nord de la Franche-Comté, nord de la Bourgogne, etc.) et en Ile-de-France, soit autant de départements dans lesquels la part de ceux qui exercent dans des communes rurales est particulièrement réduite voire nulle. Autant dire que ces médecins n'apportent pas une réponse à la crainte des déserts médicaux, alors même qu'éviter ces déserts est la raison d'être de l'agence Revitalis. À leur décharge, il faut dire qu'en Roumanie la quasi-totalité d'entre eux, du moins parmi ceux que nous avons rencontrés, vivaient en milieu urbain et que, surtout, en France, l'hôpital offre un environnement plus favorable à leur intégration. Ils arrivent en effet dans une équipe, ce qui peut faciliter les choses, alors qu'en libéral, ils sont assez isolés. À cela peuvent s'ajouter des réactions relevant a minima de l'intolérance : un des médecins interviewés installé dans une commune rurale d'Ille-etVilaine a fait état de l'ostracisme de son confrère du même village. Affirmant que l'arrivante était moins diplômée que lui, il dissuadait les patients d'aller consulter chez elle. Toutefois, dans quelques départements, la part des médecins roumains exerçant dans une commune rurale peut être relativement importante. C'est le cas de l'ensemble Lozère, Cantal, Aveyron ou, pour notre espace d'enquête, des Côtes-d'Armor (figure 1).

\section{Une présence inégale selon les statuts et les spécialités}

La répartition des médecins entre milieu rural et milieu urbain est liée de manière complexe à la répartition selon les statuts et les spécialités. La très grande majorité des praticiens roumains exercent dans le secteur public (68\% contre $32 \%$ dans le secteur privé) (figure 2) Cette prépondérance de l'exercice dans le secteur public va de paire avec celle des emplois salariés, que le praticien soit généraliste ou spécialiste. L'emploi dans le secteur privé peut relever aussi bien d'hôpitaux privés ou de service public hospitalier (PSPH) que de cabinets libéraux, dans lesquels peuvent exercer des généralistes et des spécialistes. Les variations dans la répartition par statut au niveau départemental sont surtout fonction de la présence de centres hospitalo-universitaires, du poids relatif des établissements privés, de la présence de médecins généralistes libéraux exerçant en milieu rural (figure 3).

Les centres hospitalo-universitaires accueillent aussi bien des praticiens chevronnés que des internes en formation. Les praticiens roumains y sont toujours en nombre significatif. C'est le cas 


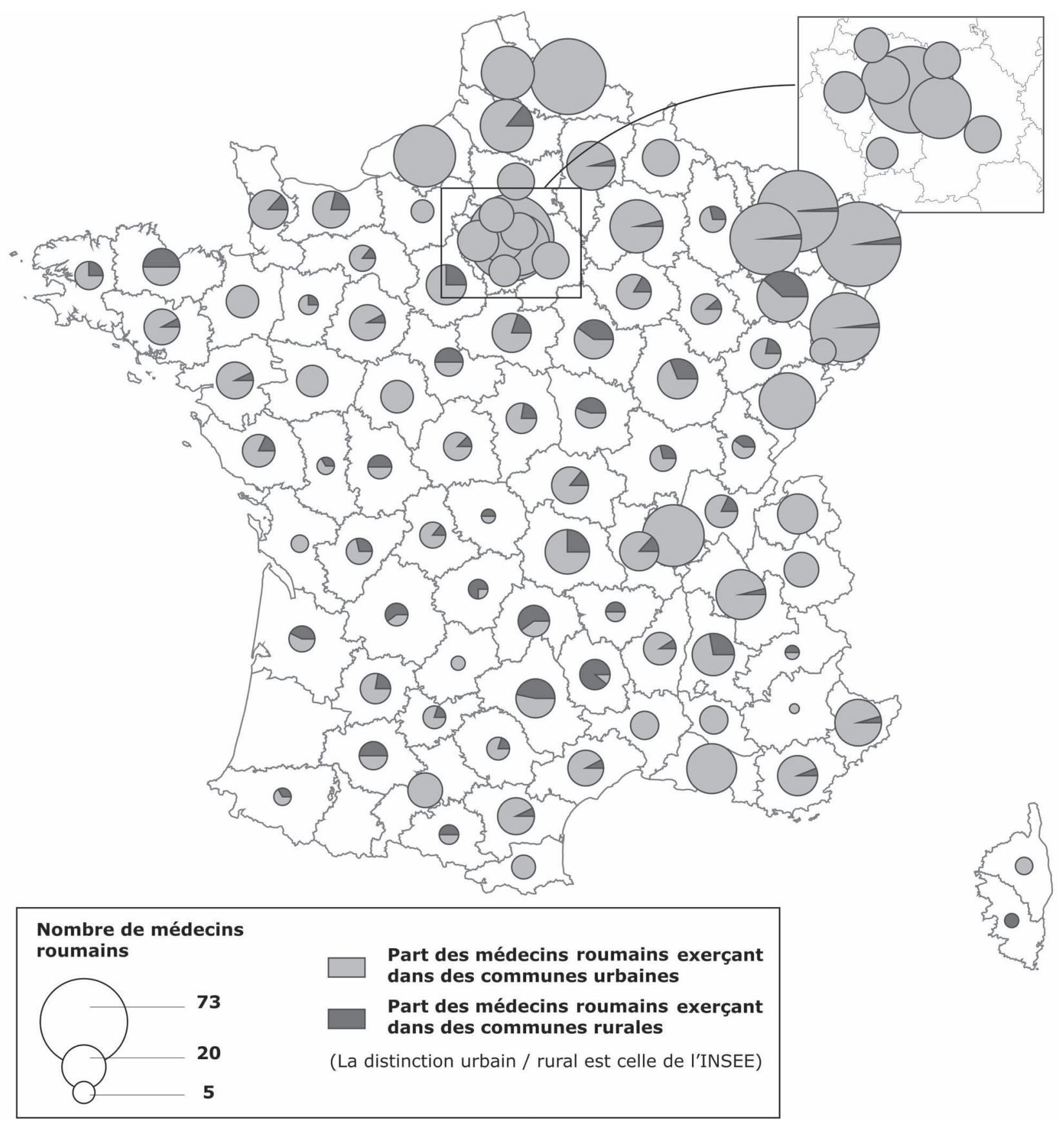

Source : DREES, 2009 - Conception : Vasilcu D., Séchet R. - Réalisation : UMR ESO

Figure 1 : Répartition des médecins roumains en France selon le type de commune (2009) Distribution of Romanians doctors in France by type of districts (2009)

des hôpitaux parisiens, Paris étant la porte d'entrée pour beaucoup de médecins roumains qui exercent en France, mais aussi, pour l'Ouest de la France, des CHU de Rennes, Brest, Caen, Nantes, Angers. Ils sont également nombreux dans des centres hospitaliers plus modestes de petites villes. Ils répon- dent alors aux difficultés de recrutement de personnels dans ces établissements, contribuant ainsi à leur survie, parfois en attente d'une fermeture de services (cas de Carhaix, Redon, Pontivy). Les établissements spécialisés dans la psychiatrie et implantés en milieu rural au XIX ${ }^{\mathrm{e}}$ siècle constituent 


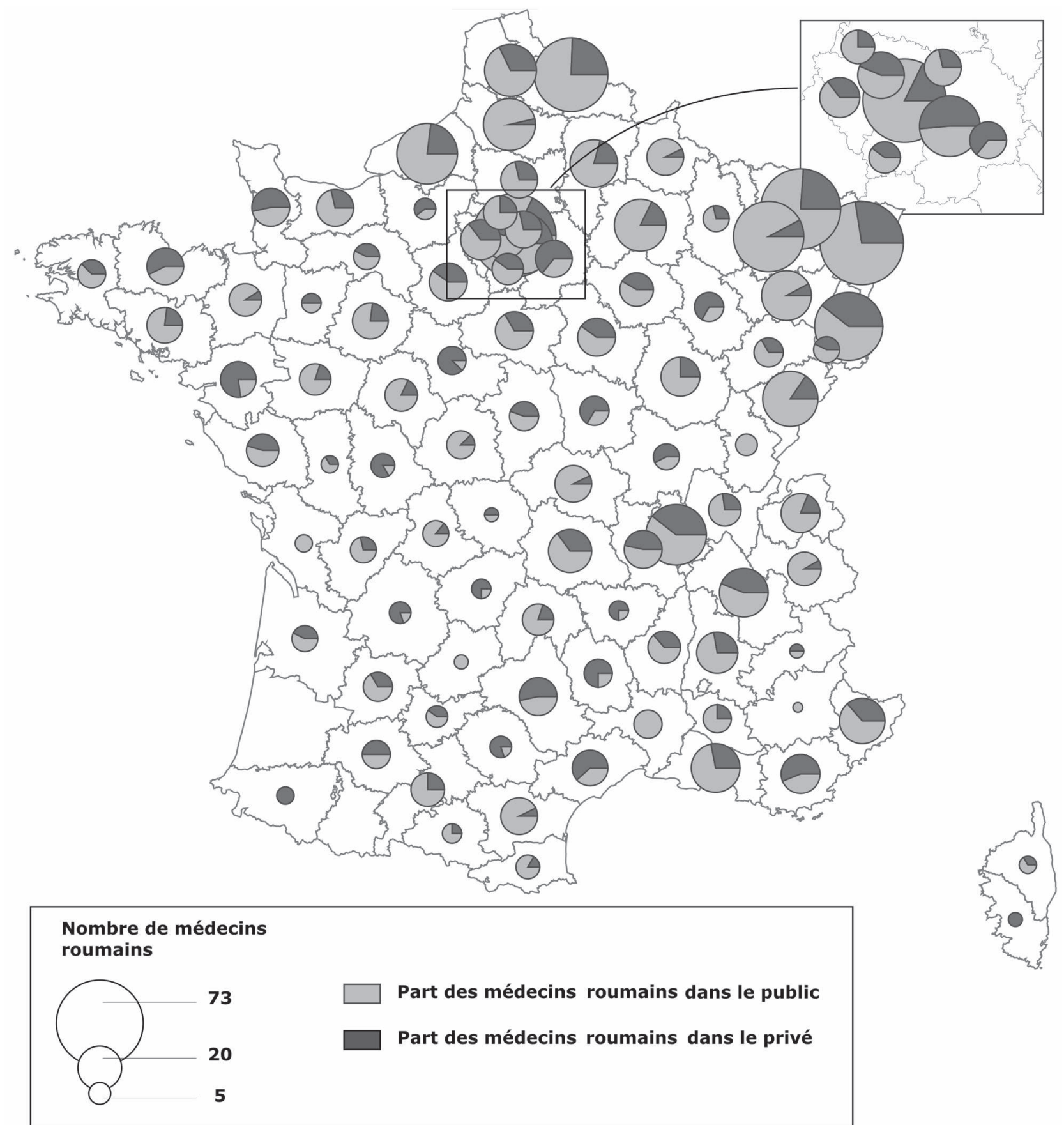

Source : DREES, 2009 - Conception : Vasilcu D., Séchet R. - Réalisation : UMR ESO

Figure 2 : Répartition des médecins roumains selon le secteur d'activité (2009) Distribution of Romanians doctors in France by sector of activity (2009)

un cas particulier. Au printemps 2010, les hôpitaux de Bégard et Plouguernével comptaient respectivement 5 et 2 psychiatres roumains. La psychiatrie est, en France, une des spécialités pour lesquelles les déficits sont les plus marqués. Et si les praticiens roumains sont présents dans toutes les spécialités, ils comptent surtout pour celles qui connaissent une forte tension : anesthésie-réanimation, radiologie, services d'urgences. 


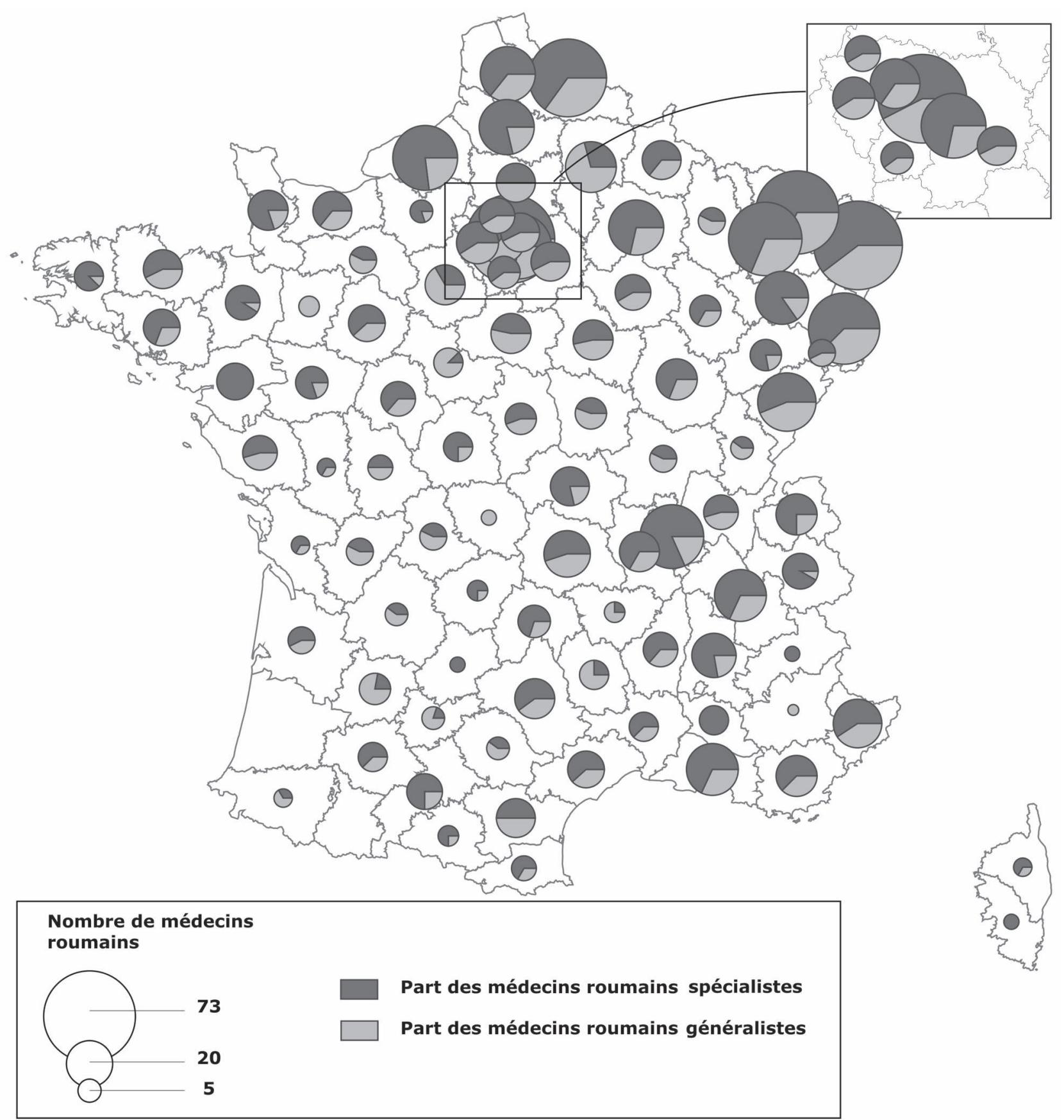

Source : DREES, 2009 - Conception : Vasilcu D., Séchet R. - Réalisation : UMR ESO

Figure 3 : Répartition des médecins roumains selon leur spécialité (2009)

Distribution of Romanians doctors in France by area of medical specialization (2009)

S'ils répondent globalement, parmi d'autres médecins étrangers, aux problèmes de démographie médicale, les médecins roumains installés en France jouent un rôle essentiel aussi bien en matière d'offre de soins dans certains espaces ruraux et les hôpitaux de leurs pôles urbains que dans les centres hospitalo-universitaires pour les spécialités les plus déficitaires, dont les services d'urgences, dans lesquelles les conditions de travail sont particulièrement difficiles. Au niveau de chacun des postes occupés, 
le bilan est assez nuancé. En effet, les médecins roumains immigrés changent fréquemment de lieux d'exercice professionnel, au risque de susciter l'agacement chez les recruteurs français (directeurs d'hôpitaux et surtout municipalités qui apprécient peu de voir repartir des médecins peu de temps après leur arrivée). L'enquête a montré qu'une fois passée la période d'arrivée et d'adaptation, ces médecins changent fréquemment de lieux d'exercice, sans que notre enquête permette de dire si cela résulte d'une certaine précarité des contrats de travail ou de stratégies personnelles.

\section{L'IMPACT DES MIGRATIONS DE MÉdecins pour la Roumanie}

La constitution d'un espace et d'un système migratoires mettant en relation l'offre de soins de Roumanie et la demande de professionnels en France consécutive au choix de gouvernements successifs de limiter les effectifs formés via la réduction du numerus clausus, participe de la progressive émergence d'un marché mondial des personnels médicaux dans lequel les mobilités répondent aussi à des objectifs personnels, familiaux et professionnels. Le système de santé roumain connaît de ce fait des difficultés de même nature que beaucoup de pays en développement.

\section{L'apport des migrations pour les migrants, leur famille et leur milieu d'origine}

Pour évaluer les apports des migrations, une distinction est à faire entre les apports microsociaux pour les migrants et leurs familles et les conséquences macro-sociales pour la Roumanie dans son ensemble.

L'apport direct des migrations dépend des pratiques de mobilités internationales après le départ de Roumanie et l'installation à l'étranger, dont la France. La médecine est une activité professionnelle qui est incompatible avec de fréquents allers-retours entre deux pays et peut difficilement s'inscrire dans la problématique des circulations migratoires. Parmi les 106 répondants à notre enquête, 68 ont fait état d'un projet d'installation durable voire définitive en France. Ce projet d'installation professionnelle est renforcé par l'affirmation de l'intérêt de la vie hors de Roumanie pour la famille. Les facteurs personnels qui relèvent de la qualité de vie et, plus particulièrement, de meilleures opportunités d'éducation et de formation pour leurs enfants ont été évoqués en tant que facteurs décisifs de la migration par 41 des 106 médecins enquêtés, tandis que 88 d'entre eux ont invoqué des facteurs socioprofessionnels comme principale motivation du projet migratoire. Ces constats incitent à penser les migrations internationales de médecins roumains comme des moments de ruptures positives dans la vie, même si, à une échelle macro, ces migrations découlent d'un ensemble de facteurs globaux, dont la dégradation des conditions d'exercice en Roumanie. Les départs hors du pays sont le moyen de réaliser un projet de vie personnel et familial (Guillaume, 2009).

Les transferts d'argent vers les pays d'origine, qui sont une des contributions des migrations internationales au développement des pays d'origine, sont à analyser sous l'angle de leur inscription dans ces projets personnels. Certes, des médecins, notamment ceux qui ont l'intention de rentrer tôt ou tard en Roumanie, transfèrent des sommes importantes pour les investir dans l'immobilier. La maison au pays est une résidence-base (Domenach et Picouet, 1987) qui facilite les mobilités temporaires à l'occasion de vacances et entretient la perspective d'un hypothétique retour. Cependant, dans la mesure où la majorité des médecins roumains émigrés se disent prêts à une installation durable ou définitive en France, ils ont tendance à investir leur argent dans ce pays plutôt qu'en Roumanie. Plus de $70 \%$ des enquêtés envoient des devises en Roumanie, mais seulement un quart de ceux qui réalisent ce type de transferts le font mensuellement. Ces envois mensuels concernent spécifiquement les médecins qui sont venus seuls ou accompagnés par leur conjoint-e et dont les enfants majeurs sont restés en Roumanie pour finir leurs études. Ils sont en général le fait de personnes qui sont en France depuis assez peu de temps. Les quelques cas observés faisant exception relèvent de situations familiales spécifiques: aide aux parents à la retraite, maladie ou incapacité de travail de membres de la famille proche. Ces transferts d'argent destinés à couvrir des besoins quotidiens ne contribuent que dans une proportion réduite à l'investissement productif et ne peuvent pas avoir un impact majeur sur le développement économique de la Roumanie. 
Conservation ou acquisition d'un bien immobilier, envoi d'argent aux proches qui sont restés au pays, mobilités temporaires à l'occasion de vacances (toutes les personnes rencontrées ont confirmé qu'elles rentrent en Roumanie au moins une fois par an) : les liens des médecins roumains émigrés avec leur pays d'origine prennent des formes classiques. L'enquête réalisée en 2010 auprès de médecins roumains installés en France laisse penser que l'impact économique de ces migrations d'élites est limité. Quant à l'impact sur le système de soins roumain, il apparaît comme étant clairement négatif. Dans la mesure où les médecins représentent une catégorie socioprofessionnelle d'importance maximale pour la santé des populations, la perte de capital humain et professionnel qui découle d'une émigration massive ne peut pas être compensée.

Les mobilités de médecins ont aujourd'hui des effets très sensibles sur l'offre de soins en Roumanie. Le phénomène migratoire qui atteint déjà une ampleur inquiétante pourrait encore s'amplifier si les difficultés économiques de la Roumanie perdurent. Le système sanitaire roumain pourrait alors connaître un collapsus lui-même générateur d'une recrudescence des départs (Vasilcu et Séchet, 2011. Au cours de l'été 2010, le Collège des Médecins de Roumanie a annoncé que $50 \%$ des médecins roumains pourraient quitter le pays dans les cinq ans à venir. S'appuyant sur les résultats d'un sondage réalisé auprès du personnel du système sanitaire roumain, une autre source a même affirmé que $70 \%$ des salariés du système de santé roumain envisageaient d'émigrer.

Dans cette même étude, les deux-tiers des répondants faisaient état d'une diminution de la qualité des services sanitaires. Le système hospitalier public roumain est confronté à la dégradation des équipements médicaux et au manque de produits sanitaires de base (médicaments, bandages, désinfectants). Comment, avec tant de départs et dans de telles conditions matérielles, offrir des prestations médicales de qualité et un égal accès aux soins pour tous, en ville comme en campagne? En partie par le recours à l'immigration : la Roumanie est à son tour devenue un pays d'immigration pour des médecins prêts à accepter les conditions d'exercice offertes par ce pays. La loi roumaine 95/2006 accorde l'exercice de la profession médicale aux médecins originaires de l'Union européenne, de Norvège et de
Suisse mais aussi aux citoyens extra-européens dont le (la) conjoint(e) ou les parents sont des Roumains et ceux qui ont terminé leurs études médicales en Roumanie et ont la citoyenneté roumaine. Dans le cadre de cette loi, un nombre croissant de professionnels de santé originaires de Moldavie et des pays arabes exercent actuellement en Roumanie, ce pays pouvant n'être qu'une étape pour ceux qui envisagent un parcours migratoire complexe ayant comme cible les pays riches de l'Union européenne.

Quoi qu'il en soit de ces nouvelles mobilités, la Roumanie risque de ne pas pouvoir atteindre les objectifs sanitaires fixés par les Nations Unies pour l'année 2015 (Drexler, 2008). L'analyse de quelques indicateurs démographiques révèle des différences considérables entre la Roumanie et les autres pays de l'UE. En 2008, l'espérance de vie à la naissance était en moyenne de 73,4 ans contre 80,6 ans pour l'Europe des Quinze. La mortalité infantile représente un autre indicateur démographique significatif pour lequel la Roumanie occupait, en 2008, le dernier rang parmi tous les pays de l'UE $(10,97 \%$ contre $3,76 \%$ en moyenne pour l'UE à 15 et $4,35 \%$ o pour l'UE à 27). Bien qu'elle ait baissé pendant la dernière décennie $(18,63 \%$ en 2000 , $16,84 \%$ en 2004 et $11,99 \%$ en 2007), la mortalité infantile se maintient à un niveau élevé, ce qui est le reflet d'un cumul de facteurs défavorables : situation économique du pays, faiblesse du niveau de vie de beaucoup de ménages, mauvaises conditions de logement, et sans doute insuffisante capacité du système de santé et des services sanitaires roumains à protéger la vie dès ses débuts.

\section{L'inscription de la Roumanie dans le marché mondial des personnels médicaux}

Pays de départ de personnels médicaux devenu pays d'immigration de professionnels en provenance de pays européens extérieurs à l'UE ou de pays plus lointains, la Roumanie est inscrite dans la recomposition des espaces migratoires marquée par une complexité accrue des flux et des efforts faits à différents niveaux (États, Union européenne, organisations internationales) pour réguler ou contrôler ces flux.

Cette complexité croissante des mobilités est à inscrire dans les débats récents sur le passage du 
«brain drain» au «brain gain » : il s'agirait, selon les organisations internationales, de faire en sorte que les pays de destination ne soient pas les seuls bénéficiaires des migrations. En fait, des ambiguïtés peuvent émerger dans l'usage fait de l'expression « brain gain » : elle peut aussi bien être lue sous l'angle du solde, en l'occurrence le gain pour les pays d'immigration (c'est incontestable pour les Etats-Unis en ce qui concerne les chercheurs) qu'être utilisée pour une lecture positive pour le pays de départ en cas de retours des élites parties quelques années à l'étranger. Pour que le départ des élites médicales puisse être vu dans cette perspective pour le moins optimiste de gains (Gaillard, 1999) qui permettraient au système sanitaire roumain d'être bénéficiaire grâce au transfert des connaissances, expériences et compétences médicales acquises dans le cadre de l'exercice professionnel à l'étranger, il faudrait que les pouvoirs publics roumains proposent et développent des politiques efficaces de retour et que les pays bénéficiaires, dont la France, ne ferment pas les yeux sur les conséquences de ces migrations pour la Roumanie!

L'argument que les migrations sélectives ont pour finalité d'encourager la venue de "personnes dont la présence est une chance pour ce pays, mais n'est pas vitale pour les pays d'origine », comme l'a dit Nicolas Sarkozy lorsqu'il était ministre de l'Intérieur et de l'Aménagement du territoire, peut être dicible, à défaut d'être 'entendable', pour ce qui concerne les recrutements de travailleurs hautement qualifiés. Il ne l'est absolument pas pour les migrations de médecins et de personnels de soins qui, par leurs missions, assurent la santé des populations et protègent la vie. Dans le cas présent, cela revient à faire payer par les Roumains l'investissement dans la formation de médecins qui vont ensuite partir en France où le nombre de places au concours d'entrée en médecine a été fixé à des niveaux inférieurs aux besoins.

Compte tenu des effets potentiellement néfastes des migrations des professionnels de santé sur les pays d'origine confrontés à de graves pénuries de personnel médical, des instruments ont été mis au point par l'OMS afin d'encourager le respect des normes éthiques pour le recrutement et l'emploi de personnels de santé venus de l'étranger. Ce n'est pas un hasard si les migrations internationales de médecins ont été au centre des débats sur les consé- quences des migrations internationales des médecins dans plusieurs sommets ou conférences internationaux récents. À l'occasion de la Conférence ministérielle européenne de l'OMS organisée à Tallinn (Estonie) en juin 2008, l'OMS a précisé que « le recrutement international des travailleurs de la santé devrait obéir à des considérations éthiques et à la solidarité internationale, et être garanti par un code de bonnes pratiques ». L'OMS, les États membres et toute une série de partenaires internationaux ont pris l'engagement politique de renforcer les systèmes de santé et, à la fin de la Conférence, ont adopté la Charte de Tallinn ${ }^{1}$ intitulée «Des systèmes de santé pour la santé et la prospérité ", Tallinn). De même, la $63^{\mathrm{e}}$ assemblée mondiale de la Santé qui s'est tenue en mai 2010 à Genève a finalisé un «code de pratique mondial pour le recrutement international des professionnels de santé ». Ce code encourage des recrutements conformes à l'éthique, tout en tenant compte de l'équilibre entre le droit de l'individu souhaitant émigrer et le souci d'équité au niveau international.

Deux extraits de la charte de Tallinn suggèrent que le gain pour les pays de départ n'est qu'un mythe, et va le rester puisqu'aucune mesure contraignante n’a été adoptée :

«Si le recrutement est bien géré, les migrations internationales de personnels de santé peuvent contribuer utilement au développement et au renforcement des systèmes de santé. Il est cependant souhaitable de fixer des principes internationaux non contraignants et de coordonner les politiques nationales en matière de recrutement international des personnels de santé pour promouvoir des cadres qui permettent de renforcer équitablement les systèmes de santé partout dans le monde, d'atténuer les effets négatifs des migrations des personnels de santé sur les systèmes de santé des pays en développement et de protéger les droits des personnels de santé. »

« Les besoins et la situation propres à chaque pays devraient être pris en considération, en particulier ceux des pays en développement et des pays à économie en transition particulièrement vulnérables aux pénuries de personnels de santé et/ou dont les moyens d'appliquer les recommandations du présent Code sont limités. Les pays développés devraient,

1. [OMS/Europe, www.euro.who.int/fr]. 
dans la mesure du possible, fournir une assistance technique et financière aux pays en développement et aux pays à économie en transition afin de renforcer les systèmes de santé, y compris sur le plan du développement des personnels de santé. »

Pour que les bénéfices de la migration des médecins soient partagés entre la Roumanie et la France, il faudrait une grande implication de la part des acteurs politiques et économiques des deux pays, ce qui n'est pas le cas. Du côté roumain, pour l'heure, la fuite des médecins n'a pas donné lieu à un programme national cohérent en faveur du retour qui ferait des médecins roumains émigrés des acteurs du développement social roumain. En fait, le gouvernement roumain n'a pas anticipé les conséquences de l'entrée de la Roumanie dans l'UE et de la reconnaissance des diplômes. Dans la Roumanie démocratique d'aujourd'hui, à l'inverse de l'ère Ceausescu où l'émigration vers l'Occident était quasiment interdite, un équilibre est à trouver entre maîtrise des flux et droit à la libre circulation qui, en raison de l'histoire récente du pays, est une revendication forte.

La connaissance des caractéristiques des médecins migrants par l'amélioration des sources disponibles sur les flux migratoires des médecins et l'attention portée aux motivations qui poussent les médecins à émigrer sont des préalables indispensables à toute action. Sans quoi les politiques susceptibles d'être adoptées sont vouées à l'inefficacité, à moins d'être coercitives et, de ce fait, porter atteintes au principe de la libre circulation et installation professionnelle des personnes. Or, à l'exception des certificats de compétence octroyés par le ministère de la Santé, le gouvernement roumain ne dispose pas de données sur le nombre et les caractéristiques des professionnels de santé qui ont émigré. Dans les débats sur la mobilité internationale des médecins et des professionnels de santé, en général, la maîtrise de l'information est déséquilibrée entre des pays qui, comme la France, disposent de statistiques relativement précises (via à la fois le gouvernement et l'Ordre des médecins) et des pays qui, comme la Roumanie, n'ont que des données lacunaires et peu fiables. Les autorités roumaines soutiennent aujourd'hui les actions visant à rapprocher l'ensemble des médecins roumains installés en France : une première rencontre en vue de constituer une association a été organisée à Paris le 29 mai 2010 à Paris avec l'aide de l'ambassade de Roumanie en France. Les objectifs d'une telle initiative n'ont pas été précisés mais on peut supposer que les logiques de maîtrise des informations, de contrôle social et de pérennisation des liens avec la Roumanie pour favoriser un éventuel retour ultérieur n'en sont pas absentes.

\section{Conclusion}

La réduction des numerus clausus dans les facultés de médecine, et donc, avec un décalage dans le temps d'une dizaine d'années, celle des effectifs de nouveaux médecins, est à l'origine de la crise de la démographie médicale dont le gouvernement a reconnu l'ampleur au début des années 2000. Bien que le recours aux migrations n'apparaisse pas explicitement dans les solutions envisagées pour maintenir l'offre de soins dans un contexte d'augmentation des besoins inhérent au vieillissement de la population et, il faut bien le dire, de désengagement dans l'offre de formation au cours des premières années d'études de médecine, les effectifs de médecins étrangers exerçant en France ont augmenté. Le contexte européen et roumain a été favorable au départ de médecins roumains qui sont maintenant très présents dans le paysage sanitaire français. Sollicités face à la crainte de la désertification des campagnes françaises, ils répondent surtout aux besoins des hôpitaux pour certaines spécialités particulièrement déficitaires.

Pour la Roumanie, l'exode de ses médecins constitue la perte d'un élément vital pour le développement social du pays, une perte plus grave que celle de toute autre catégorie professionnelle en raison de ses conséquences pour l'avenir de la population roumaine. L'émergence et le maintien des discontinuités en matière de richesse dans une Europe à plusieurs vitesses marquée par de forts clivages Est-Ouest (Vandermotten, 2006) risquent d'amplifier dans les années à venir le brain drain médical roumain. Pays pauvre à l'échelle de l'Europe, la Roumanie va payer chèrement la liberté de circulation, d'installation et de travail de ses citoyens. Paradoxalement, cette liberté acquise après 2007, en dépit des avantages qu'elle comporte, va dresser des obstacles économiques et sociaux qui seront difficiles à surmonter. Comment et dans combien 
d'années la Roumanie pourra-t-elle assurer des conditions de vie et d'exercice de la profession comparables à celles des pays occidentaux, et ainsi espérer freiner la vague de départs et, pourquoi pas, inciter les médecins émigrés à revoir leurs projets?

\section{Bibliographie}

Attal-Toubert K., Vanderschelden M., 2009. La démographie médicale à l'horizon 2030 : de nouvelles projections nationales et régionales, DREES, Études et Résultats, $n^{\circ} 679,49$.

Bessiere S., Breuil-Genier P., Darrine S., 2004. La démographie médicale à l'horizon 2025 : une actualisation des projections au niveau national. DREES, Études et Résultats, $\mathrm{n}^{\circ} 353,12 \mathrm{p}$.

Cash R., Ullman P., 2008. Projet OCDE sur la migration des professionnels de santé : le cas de la France, OCDE, Health Working Paper, n 36, 117 p.

Conseil National de l'Ordre des Médecins (CNOM), 2009 (4e édition), L'Atlas de la démographie médicale en France, [http://www.conseil-national.medecin.fr/system/files/ atlas2009_0.pdf].

Constant F., 2009, Pour une gouvernance mondiale des migrants, in Jaffrelot C., Lesquesne C. (dir.), L'enjeu mondial. Les migrations, Presses de Sciences Po - L'Express, Paris, p. 291-306.

Cortes G., Faret L., 2009, Les circulations transnationales : lire les turbulences migratoires contemporaines, Armand Colin, Paris, 248 p.

Darrine S., 2002. Un exercice de projection de la démographie médicale à l'horizon 2020 : les médecins dans les régions et par mode d'exercice, DREES, Etudes et résultats, n ${ }^{\circ}$ 156, $12 \mathrm{p}$.

Docquier F., Rapoport H., 2007. L'immigration qualifiée, remède miracle aux problèmes économiques européens, Reflets et perspectives de la vie économiques, 2007/1, Tome XLVI, p. 95-111.

Domenach H., Picouet M., 1987. Le caractère de réversibilité dans l'étude de la migration, Population, n 3, p. 469-484.

Drexler A., 2008. Le défi du recrutement des médecins à diplôme étranger dans les hôpitaux publics, Rennes, Mémoire de l'École des hautes études en santé publique (EHESP),
70 p. + annexes, [http://ressources.ensp.fr/memoires/2008/ edh/drexler.pdf].

FARET L., 2003. Les territoires de la mobilité. Migration et communautés transnationales entre le Mexique et les États-Unis, CNRS Éditions, Paris, 351 p.

Gaillard A-M., Gaillard J., 1999. Les enjeux des migrations scientifiques internationales. De la quête du savoir à la circulation des compétences, L'Harmattan, Paris, 234 p.

Guillaume J.-F., 2009. Les parcours de vie, entre aspirations individuelles et contraintes structurelles, Informations sociales, $\mathrm{n}^{\circ} 156$, p. 22-30.

Guimah-Brempong K., Wilson M., 2004. Health human capital and économic growth in Sub-SaHaran African AND OECD COUnTRIEs, The Quarterly Review of Economics and Finance, vol. 44, n² 2, p. 296-320.

Hily M-A., Berthomière W., Mihaylova D., 2004. La notion de "réseaux sociaux" en migration. Hommes et Migrations, $\mathrm{n}^{\circ} 1250$, p. 6-12

Mayer J.-B., 2009. La percolation mondiale des compétences, in Jaffrelot C. et Lesquesne C., L'enjeu mondial. Les migrations, Presses de Sciences Po, p. 245-252.

SALt J., 2005. Evolution actuelle des migrations internationales en Europe, Rapport pour le Conseil de l'Europe, janvier 2005, [http://www.coe.int/t/dg4/linguistic/Source/SALT_FR.pdf].

Secrétariat Général du Comité Interministériel de Contrôle de l'Immigration (SGCICI), 2008. Les orientations de la politique de l'immigration, La Documentation Française, (5 rapport au Parlement établi en application à l'article L. 111-10 du Code de l'entrée et du séjour des étrangers et du droit d'asile), 236 p.

Tandonnet M., 2003. Migrations. La nouvelle vague, Paris, L'Harmattan, $232 \mathrm{p}$.

VAndermotten C., 2006. La géographie de l'Europe : un demisiècle de mutations... et après?, in Decroly J.- M., NicoLAÏ H., Mutations des territoires dans le monde à l'aube du $\mathrm{XXI}^{\mathrm{e}}$ siècle, Paris, l'Harmattan, p. 13-57.

VAsilcu D., SÉchet R., 2011 . Vingt ans d'expérience migratoire en Roumanie postcommuniste, Espace, Populations, Sociétés, $\mathrm{n}^{\circ} 2$, p. 43-55.

Weber S., 2007. Nouvelle Europe, nouvelles migrations. Frontières, intégration, mondialisation, Éditions du Félin, 118 p.

Weber S., 2009. D'un rideau de fer à l'autre : Schengen et la discrimination dans l'accès à la mobilité migratoire, Geocarrefour, Vol. 84, n³, p. 163-171. 